\title{
Regulation of Biologicals: Indian Perspective
}

\section{${ }^{1}$ Surinder Singh, ${ }^{2}$ Akanksha Bisht}

How to cite this article: Singh S, Bisht A. Regulation of Biologicals: Indian Perspective. Int J Res Foundation Hosp Healthc Adm 2015;3(2):135-136.

\section{Source of support: Nil}

Conflict of interest: None

\section{INTRODUCTION}

The annual turnover of the Indian pharmaceutical industry is estimated to be about ₹ $128,044.291$ crores during the year 2013 to 2014 . The Indian biotech industry as per the data available is of the size of ₹ 25,165 crore approximately, of which $63 \%$ of the revenue is contributed by the biopharma sector, i.e. ₹ 15,853 crore. The strength of biopharma sector is evident from the fact that Indian biopharma companies are not only meeting the domestic requirement of the vaccines but also exporting these to more than 150 countries across the globe. Indian vaccine industry has occupied an important niche in the manufacturing of EPI vaccines in the last decade and is one of the major suppliers to United Nations (UN) agencies of pre-qualified vaccines.

This is a testament of impeccable credentials with respect to safety, quality and efficacy of the vaccines produced by Indian companies. Now, the Indian biopharma companies are venturing into the areas of development of tetravalent dengue vaccine, oral cervical cancer vaccine including vaccines for neglected tropical diseases, such as chikungunya and kala-azar. The regulations play a very vital role in the growth of this sector.

\section{REGULATION OF BIOLOGICALS}

The import and manufacture of drugs including biologicals are regulated under the provisions of drugs and cosmetic act 1940 and drugs and cosmetic rules 1945.

\footnotetext{
${ }^{1}$ Director, ${ }^{2}$ Officer-in-Charge

1,2 Hemovigilance Program of India Cell, National Institute of Biologicals, Ministry of Health and Family Welfare, Government of India, Noida, Uttar Pradesh, India
}

Corresponding Author: Akanksha Bisht, Officer-in-Charge Hemovigilance Program of India Cell, National Institute of Biologicals, Ministry of Health and Family Welfare, Government of India, A-32, Sector-62, Noida-201309, Uttar Pradesh, India e-mail: akankshabisht83@gmail.com
The import and manufacture of biologicals without a license is an offence under the act. As such there is no separate definition of biosimilar in the act and rules, however, CDSCO and department of biotechnology published guidelines on regulation of similar biologic in 2012 which gives detailed account of regulatory expectations for the licensing of recombinant DNA products.

Biologicals in India include both vaccines as well as recombinant DNA products which are considered as new drugs as per the Drugs and Cosmetic Rules 1945. These products require new drug approval from central licensing authority popularly known as Drugs Controller General of India (DCGI) before grant of license to manufacture in the country by State Licensing Authorities (SLAs).

The drugs and cosmetic rules provide specific information to be furnished by the applicant for approval of new drug as specified in Form 44 read with schedule Y. The Schedule Y gives detailed guidelines on conduct of preclinical and clinical studies and data required in respect of manufacture and quality control.

As per Indian guideline, one repeat dose toxicity study is sufficient to demonstrate comparability in relevant animal model. In case of in vivo toxicity studies, at least one repeat dose toxicity study in a relevant species is required to be conducted for appropriate duration generally not less than 28 days with 14 days recovery period. Clinical studies usually phase 3 is also required as per Indian guidelines for approval of biologicals. Generally for vaccine, comparative phase 3 clinical trial in healthy volunteers is required. In cases where vaccines are meant for infants descending trial is needed to prove safety as well as efficacy. In case of biopharmaceuticals, the pharmacokinetics studies/pharmacodynamics studies, confirmatory safety and efficacy studies and safety and immunogenic data may be required for new drugs approval. For novel biologics, the firm is required to do a phase I, II and III clinical trial before marketing authorization is granted to the firm.

The similar biologics/novel biologics project work starts with either 'clone/strain development or clone/ strain import', for which permission is needed from RCGM or DCG (I) as the case be. In case of recombinant product, the firm starts by submitting either clone development strategy or existing data for the clone to be 
imported for IBSC approval. All applications submitted to RCGM need to be accompanied with an IBSC approval for the same. The clone development or import application is reviewed in RCGM meeting after which the clone development or clone import approval is granted to the firm. Similarly, application for 'carry-out-research' is made to RCGM after IBSC approval. The 'carry-outresearch' application is reviewed in RCGM meeting after which the 'carry-out-research' approval is granted to the firm. The above two applications ('clone development or clone import' and 'carry-out-research') can be made to RCGM at the same time.

The firm also applies for NOC for Form 29 to DCGI. Prior to granting NOC for Form 29 approval, DCGI requests for joint inspection of the facilities to state FDA and CDSCO. On successful completion of joint inspection DCGI grants NOC for Form 29 approval, based on which Form 29 is granted by state FDA.

After receiving above referred approvals from RCGM, DCGI and state FDA, the firm develops/ imports the clone/strain and initiates the analytical and process development activities. Once the process is frozen, three consistency batches are taken and product characterization is completed, the firm applies for approval to conduct preclinical toxicity studies to IBSC in case of recombinant products, however, in case of vaccine no such approval in required.

Once the preclinical studies are completed the firm applies to DCG (I) for grant of approval of clinical trial proposal in Form 44 as per CDSCO format for clinical trial application along with $\mathrm{CT}$ protocol. Drugs controller general of India reviews the $\mathrm{CT}$ application for CMC and nonclinical and the CT protocol. Drugs controller general of India forward the $\mathrm{CT}$ protocol to subject expert committee (SEC) for review and comments. Subject expert committee meeting is conducted where the firm does a presentation on the $\mathrm{CT}$ protocol and design to obtain CT Protocol approval from SEC. Once the SEC gives favorable comments, the file is forwarded to technical committee as well as apex committee. Once technical committee and apex committee approves the CT protocol, DCGI grants $\mathrm{CT}$ permission to the firm.

After receiving CT permission from DCGI, the firm initiates the clinical trial. Once the clinical trial is completed, the firm applies for marketing approval to DCGI in Form 44 as per CDSCO application format for marketing authorization along with $\mathrm{CT}$ report. Drugs controller general of India reviews the marketing application for CMC and non-clinical and clinical sections, i.e. CT report, and forwards the $\mathrm{CT}$ report to
SEC for review and comments. Subject expert committee meeting is conducted where the firm has to defend the CT report and results to obtain approval/favorable comments from SEC. Once the SEC gives favorable comments, DCGI grants marketing approval [Form 46A (for drug substance) and Form 46 (for drug product)] to the firm.

After obtaining Form 46A (for drug substance) and Form 46 (for drug product), the firm applies in Form 27D for Form 28D (manufacturing license) for both drug substance and drug product to SLA. In case the site has not been inspected with experts, joint inspection involving officers of Central Drugs Standard Control Organization, expert and officers of SLA is carried out to verify the firms compliance to GMP and product specific requirements. The report of the inspection is reviewed by the SLA and if satisfied, SLA sends the Form 28D to DCGI for approval. Once the approval is granted by DCGI on Form 28D, SLA grants Form 28D to the firm.

Once Form 28D is granted the firm manufactures commercial batches, ensure consistency of the batches produced and then launches the product in Indian market.

\section{QUALITY CONTROL OF BIOLOGICALS}

Biologicals are complex molecules difficult to characterize and standardize given to inherent variability from batch to batch and their test/analysis requires highly specialized facilities and domain expertise supported by an animal facility.

There are two laboratories at the national level, i.e. (i) central drugs laboratory, Kasauli, Himachal Pradesh; (2) National Institute of Biologicals, Noida, Uttar Pradesh, which are involved in quality control of biologicals.

The central drugs laboratory kasauli does the quality control testing and lot release for vaccines whereas National Institute of Biologicals is notified as Central Drugs Laboratory under Drugs and Cosmetic Act and Rules for various biologicals, i.e. (a) blood grouping reagents, (b) diagnostic kits-HIV/HBsAg/HCV, (c) blood products-human albumin, human norman immunoglobulin-IV/IM, human coagulation factor VIII, human coagulation factor IX, plasma protein factor, fibrin sealant kit, anti-inhibitor coagulation complex, (d) recombinant DNA products-r-insulin and insulin analogs, r-erythropoietin (EPO), r-granulocyte colony stimulating factor (G-CSF) and (e) glucose test strips and fully automated analyzer based glucose reagents.

Thus, the Indian regulatory system is robust and dynamic to meet the challenges thrown up by newer biologicals being launched or developed in the country from time to time. 